\title{
Study on the strength loss of Multi-hop HF Radio Propagation
}

\author{
Lingxiao Liu ${ }^{1}$, Tian Lu ${ }^{1}$, Mingxue Gong ${ }^{1}$, Wuyu Zhang ${ }^{1}$ \\ 1.School of Civil Engineering, Qinghai University, Xining Qinghai, 810016, China
}

\begin{abstract}
The reflections of high frequency (HF) radio waves between ionosphere and earth's surface make long-distance information transmission possible. In this paper, the propagation process of radio signals was analyzed and the ionosphere was simplified. Considering the strength loss of signals that occurs in the travelling process and at the reflection points, two pairs of differential equations and integral equations were established to simulate the strength variations of HF radio waves and noises. A different equation of SNR was also developed, which utilized the failure threshold of signal-noise-ratio (SNR) as a criterion to evaluate the effectiveness of signals. Meanwhile, the pace of SNR attenuation was simulated when reflections happens on calm ocean, turbulent ocean, smooth terrain and rugged terrain.
\end{abstract}

\section{Introduction}

On high frequencies (HF, defined to be 3-30 MHz), radio waves can travel long distances (from one point on the earth's surface to another distant point on the earth's surface) by multiple reflections off the ionosphere and off the earth. The characteristics of the reflecting surface determine the strength of the reflected wave and how far the signal will ultimately travel while maintaining useful signal integrity.

\section{Modeling process}

\subsection{Physical model simplification}

Considering the propagation of HF radio waves between the ionosphere and the earth as a process from one point to another distant point on the earth's surface, the assumption was made that both the ionosphere and the earth's surface can be considered as two curved surfaces which do not intersect (Fig. 1). In this way, the propagation of HF radio waves can be simplified to a process in which the waves travel up and down at a certain angle between the two curved surfaces.

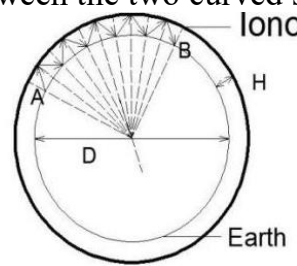

Fig.1Radio waves reflections off the and off the earth.

\subsubsection{Loss in Free Space}

Loss in free space refers to the phenomenon that the radio wave energy spreads naturally with the propagation distance. The free space presented here is an ideal case for an infinitely large vacuum environment where the medium is homogeneous and isotropic. The conductivity, the relative permittivity and the relative permeability are $0^{[1]}$ respectively.

the strength loss in free space $\mathrm{q}_{\mathrm{f}}$ is denoted by:

$$
q_{f}=\left(\frac{4 \pi x}{\lambda}\right)^{2} \text { (1) }
$$

In the formula, $\lambda$ is the wavelength, $D$ is the distance between two adjacent reflection points of earth's surface.

\subsubsection{Loss through the Medium}

When radio waves propagate through the medium, a certain amount of loss occurs. Here, the wave impedance was used to calculate. In particular, for a plane wave travelling through empty space, the wave impedance is equal to the impedance of free space. The symbol $\mathrm{Z}$ is used to represent the impedance of free space and it is expressed in units of ohms.

In free space the wave impedance of plane waves is:

$$
Z_{0}=\sqrt{\frac{\mu_{0}}{\varepsilon_{0}}}
$$

Where $\varepsilon_{0}$ is the permittivity constant in free space and $\mu_{0}$ is the permeability constant in free space.

$$
Z_{0}=\mu_{0} c_{0}=4 \pi \times 10^{-7} H / m \times 299,792,458 m / s=377 . \Omega^{[2]}
$$

\subsection{Factors that affect signal attenuation}




\subsubsection{Loss at Reflection Points of earth's Surface}

Loss at reflection points of earth's surface is generated when the radio waves are reflected off the earth's surface at a certain point, which occurs only when the number of hops is equal or greater than two. Here, the reflection coefficient of earth's surface $\rho$ was introduced to calculate this kind of loss, using the formula derived by Ament (1953) ${ }^{[3]}$, the equation is as follows:

$$
\rho=\exp \left[-2 \frac{2 \pi \sigma_{h} \sin \varphi}{\lambda}\right](2)
$$

Where, $\lambda$ is the wavelength, $\sigma_{h}$ is the root mean square of wave height, $\varphi$ is the incident angle of the radio wave.

The incident angle of the radio wave $\varphi$ ranges from 0 to $\pi / 2$. And the wave height adopted in this paper ranges from 0 to $15 \mathrm{~m}$. The Fig. 2 below is the three-dimensional image simulation of equation (2) using MATLAB ${ }^{[4]}$ :

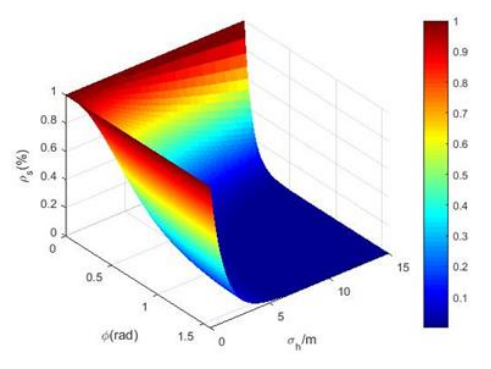

Fig.2 The three-dimensional relationship between $\rho, \sigma_{h}$ and $\varphi$

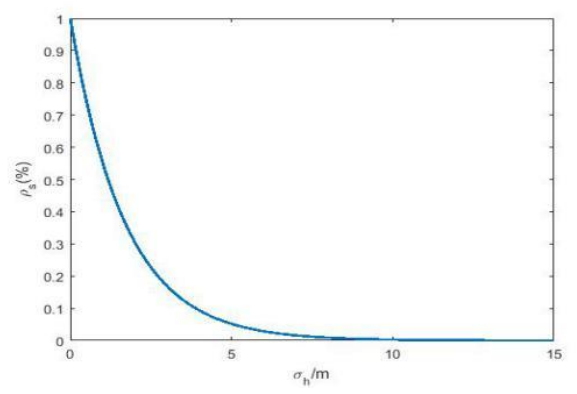

Fig.3 When $\varphi=\pi / 4$, the relationship of $\rho$ changes with the $\sigma_{h}$

When $\varphi=\pi / 4$, the relationship between the reflection coefficient $\rho$ and the root mean square of wave height $\sigma_{h}$ is shown in Fig. 2.

\subsubsection{Loss at Reflection Points of lonosphere}

The radio waves reflection off ionosphere is similar to that off the earth's surface, and its reflection coefficient can be expressed as (2).

\subsection{Mathematical modeling}

From the four factors above, it was assumed that the loss in free space and the loss through the medium are the strength loss of radio waves in straight-line transmission, and that the signal strength decreases as the transmission distance increases. Here, synthetic attenuation coefficient $\mu$ was used as a parameter to represent their impact. According to the literature, it was assumed that the $\mu$ has a negative exponential distribution.

The loss at reflection points of ionosphere and earth's surface are the loss of radio waves when it reaches the point of reflection of the surface. The strength loss of radio waves increases as the number of hops increases, and the number of hops is related to transmission distance. The synthetic reflection loss coefficient $\eta$ was used as a parameter to represent their impact.

Here, $\mathrm{q}$ is the signal strength of the radio wave, $\mathrm{x}$ is the signal transmission distance and $d x$ is the slight increment of the signal transmission distance [5]. According to the radio wave theory, the following formula was built:

$$
q(x+d x)=q(x) e^{-\mu(x) d x}-q(x) \eta(x)
$$

When the radio waves are on the reflective surface, the above formula expands to series, retaining the first-order items, so we have:

$$
q(x+d x)=q(x)-q(x) \mu(x) d x-q(x) \eta(x)
$$

or

$$
q(x+d x)-q(x)=-q(x) \mu(x) d x-q(x) \eta(x)(3)
$$

The above equation is the difference equation for calculating the radio wave signal strength $q(x)$. Since the signal increment $d x$ is a small amount, then equation (3) can be written as:

$$
d q=-q(x) \mu(x) d x-q(x) \eta(x)(4)
$$

Formula (4) is written in an integral form:

$$
q(x)=q\left(x_{0}\right)-\int_{x_{0}}^{x} q(z) \mu(z) d z-\sum_{k=1}^{N(x)} q\left(x_{k}\right) \eta\left(x_{k}\right)(5)
$$

Where, $N(x)$ is the number of reflections of the radio wave, $q\left(x_{0}\right)$ indicates the initial signal strength and $x_{k}$ represents kth hop.

Equation (5) is the basic equation of model and it varies when $\mu$ or $\eta$ is set with a special value, which can be expressed as follows:

Table 1 Model representation under special circumstances

\begin{tabular}{ccc}
\hline$\mu$ & $\eta$ & The basic equation of the model \\
\hline constant & 0 & $q(x)=q\left(x_{0}\right) e^{-\mu x}$ \\
$0 \quad$ variable & $q(x)=q\left(x_{0}\right)-\sum_{k=1}^{N(x)} q\left(x_{k}\right) \eta\left(x_{k}\right)(6)$ \\
constant variable & $q(x)=\sum_{k=1}^{N(x)+1} q\left(x_{k-1}\right) e^{-\mu\left(x_{k}-x_{k-1}\right)}$ \\
& $-\sum_{k=1}^{N(x)} q\left(x_{k}\right) \eta\left(x_{k}\right)$
\end{tabular}




\subsection{The value of the parameter}

\subsubsection{Synthetic attenuation coefficient $\mu$}

The wave impedance of radio waves through medium is about $377 \Omega$ and the loss of strength in free space can be calculated from formula (1), therefore the strength loss of unit distance in free space can then be expressed as below:

$$
\frac{q_{f}}{x}=\frac{16 \pi^{2} x}{\lambda^{2}}
$$

Where, $\lambda$ is the wavelength, $\mathrm{x}$ is the signal transmission distance.

As it is assumed that the signal's synthetic attenuation coefficient $\mu$ has a negative exponential distribution, then it can be represented as:

$$
\mu(x)=\exp [-377]+\exp \left[-\left(\frac{16 \pi^{2} x}{\lambda^{2}}\right)\right](7)
$$

\subsubsection{Synthetic Reflection Coefficient $\boldsymbol{\eta}$}

In section 2.2.3, the expression (2) of the earth's surface reflection coefficient $\rho$ was obtained. Taking into account the instability of different reflection surfaces' states, the correction coefficient $\eta_{0}$ was introduced on the basis of formula (2) and the synthetic reflection coefficient $\eta$ was obtained.

$$
\eta=1-\rho=1-\eta_{0} \cdot \exp \left(-2 \frac{2 \pi \sigma_{h} \sin \varphi}{\lambda}\right)
$$

In the formula, $\eta_{0}$ is the correction coefficient, $\lambda$ is the wavelength, $\sigma_{h}$ is the root mean square wave height, $\varphi$ is the incident angle of the radio wave.

\subsubsection{The number of hops $N$}

The distance between two adjacent hops on the ground was considered as a micro-segment, and the earth's surface was considered as a plane. The height from that layer to the ground is 60 to $90 \mathrm{~km}$. Here, the vertical height of ionosphere was valued $80 \mathrm{~km}$. According to geometric relationship, it can be concluded that the distance between two adjacent reflection points on the ground $d$ is:

$$
d=2 \times \frac{80}{\tan \theta}=\frac{160}{\tan \theta}
$$

In the formula, $\theta$ is the angle between the transmission direction of the radio wave and the reflection surface.

Then the number of hops on the $x$ distance transmitted by the radio waves is:

$$
N(x)=\left[\frac{x \cos \theta}{d / 2}\right]=\left[\frac{2 x \cos \theta}{160 / \tan \theta}\right]=\left[\frac{x \sin \theta}{80}\right] \text { (9) }
$$

\subsection{The maximum number of hops that can be reflected when the signal intensity is attenuated to failure}

$\mathrm{S} / \mathrm{N}$ ratio ${ }^{[6]}$ is defined as the ratio of signal power to noise power, often expressed in decibels. The formula is as follows:

$$
S N R=\frac{q}{z}(10)
$$

It is believed that when the signal to noise ratio is reduced to less than $10 \mathrm{~dB}$, the signal can be identified as a signal failure and the signal cannot be recognized.

Considering the impact of noise growth:

$$
\begin{aligned}
z(x+d x)= & z(x) e^{-\mu(x) d x}-z(x) \eta(x) \\
& +q(x) v(x) d x+q(x) \tau(x)
\end{aligned}
$$

Where, $v(x)$ is the proportional coefficient to the signal strength, $\tau(x)$ is the proportional coefficient between the reflective point noise intensity increment and the signal strength.

The above formula expands to a series and retaining first-order items:

$$
\begin{aligned}
z(x+d x)-z(x) & =-z(x) \mu(x) d x-z(x) \eta(x) \\
& +q(x) v(x) d x+q(x) \tau(x)
\end{aligned}
$$

Then equation (11) is the difference equation for calculating $z(x)$. Since $d x$ is small, the above equation can be written as:

$$
\begin{aligned}
d z= & -z(x) \mu(x) d x-z(x) \eta(x) \\
& +q(x) v(x) d x+q(x) \tau(x)
\end{aligned}
$$

Writen in an integral form as:

$$
\begin{aligned}
z(x) & =z\left(x_{0}\right)-\int_{x_{0}}^{x} z(u) \mu(u) d u+\int_{x_{0}}^{x} q(u) v(u) d u \\
& -\sum_{k=1}^{N(x)} z\left(x_{k}\right) \eta\left(x_{k}\right)+\sum_{k=1}^{N(x)} q\left(x_{k}\right) \tau\left(x_{k}\right) \quad(12)
\end{aligned}
$$

Equation (12) is the basic model of noise strength.

When $\mu=0, v=0$, the solution of the basic equation is $z(x)$ :

$$
z(x)=z\left(x_{0}\right)-\sum_{k=1}^{N(x)} z\left(x_{k}\right) \eta\left(x_{k}\right)+\sum_{k=1}^{N(x)} q\left(x_{k}\right) \tau\left(x_{k}\right)
$$

The equation contains two parts: the attenuation of noise during reflection and the increase of noise caused by electromagnetic interference.

In (12), the following formula was used to calculate the noise coefficient $\tau$.

$$
\tau=1-\tau_{0} \rho=1-\tau_{0} \times \exp \left[-2 \frac{2 \pi \sigma_{2 h} \sin \varphi}{\lambda}\right]
$$

Then MATLAB was used to simulate the total function model. The root mean square wave height was valued $2 \mathrm{~m}$, the angle of incidence of radio waves was valued 45 degrees, $\eta_{0}=0.4 ; \tau_{0}=0.05 ; \lambda=15 \mathrm{~m}$. The initial intensity of the signal is $50 \mathrm{dBm}$ and Ionospheric wave height is $50 \mathrm{~m}$. The calculated results are shown in Table 2: 
Table 2 The signal strength in different ground, different hop number

\begin{tabular}{ccccc}
\hline $\begin{array}{c}\text { the number } \\
\text { of hops }\end{array}$ & $\begin{array}{c}\text { clam } \\
\text { ocean }\end{array}$ & $\begin{array}{c}\text { Turbulent } \\
\text { ocean }\end{array}$ & $\begin{array}{c}\text { Smooth } \\
\text { terrain }\end{array}$ & $\begin{array}{c}\text { rugged } \\
\text { terrain }\end{array}$ \\
\hline 1 & 27.0805 & 21.3671 & 21.4958 & 18.9712 \\
2 & 20.1490 & 14.4356 & 14.5643 & 12.0397 \\
3 & 16.0944 & 10.3810 & 10.5097 & 7.9851 \\
4 & 13.2176 & 7.5042 & 7.6329 & 5.1083 \\
5 & 10.9861 & 5.2727 & 5.4014 & 2.8768 \\
6 & 9.1629 & 3.4495 & 3.5782 & 1.0536 \\
\hline
\end{tabular}

From Table 2, it can be concluded that the number of hops of radio waves on the ocean surface is greater than the number of hops on the surface of the land, that is, the loss of the signal strength of the ocean surface is less than the loss of signal strength on the land surface, and the loss of signal strength over the rugged terrain is greater than the loss of the signal strength of the smooth terrain.

\section{Conclusions}

(1) The characteristics of the reflecting surface determine the strength of the reflected wave and how far the signal will ultimately travel while maintaining useful signal integrity.

(2) The number of hops of radio waves on the ocean surface is greater than the number of hops on the surface of the land, that is, the loss of the signal strength of the ocean surface is less than the loss of signal strength on the land surface, and the loss of signal strength over the rugged terrain is greater than the loss of the signal strength of the smooth terrain.

\section{Acknowledgments}

This study was supported by S\&T Foundation Platform of Qinghai Province (Grants No. 2018-ZJ-T01),The Cooperation Program of Qinghai Province (Grants No. 2017-HZ-804).

\section{References}

1. Yuan Xiao-bo. Propagation loss prediction and field strength prediction of short-wave sky-wave [J]. Information and Communication

$$
\text { Co,2013(5):11-12. }
$$

2. https://en.wikipedia.org/wiki/Wave_impedance Wiki pedia, Wave impedance.

3. Ament,W.S. Toward a theory of reflection by a rough surface[M].Proceedings IRE 41:142-146.

4. Liu Hao, Ha Jing. Completely Self-taught in MATLAB R2012a [M].2013:368-378.

5. Han Zhonggeng. Mathematical Modeling Method and Application[M].2009:55-68.

6. Xiang Guangming. the Theoretical Limit of Signal-to-noise Ratio of low-light-level Image
Intensifier[J].Applied Optics,2008(29):24-26. 